\title{
PUBLIC HEALTH PROBLEMS
}

\section{of Migrant Workers and Their Families}

\author{
A. L. CHAPMAN, M.D.
}

$\mathrm{D}^{\mathrm{I}}$

IFFERENT PEOPLE look at migrant workers through different eyes. Local physicians and hospital administrators become aware of migrants when they come to the doctor's office or to the hospital in an emergency. Sanitarians are acutely aware of the substandard housing and sanitation facilities provided many migrants. Public health nurses see the diarrhea, impetigo, worms, and malnutrition, particularly, of migrant children.

Each spring an army of migrants take their children out of school, pack up their household belongings, start up their trucks and jalopies, and head for the promised land.

Some have firm contracts with growers and know where they are going to work; others are never sure where they will find work. They simply follow the crops from State to State, locality to locality, gambling on the weather, the crops, and state of disrepair of their tires.

Some work only a short period of time each summer and cover only a few hundred miles in their wandering. Others are on the road for 5 or 6 months and span thousands of miles. A few travel from area to area and have no permanent home.

It has been estimated that more than 8,000 migrants come to Pennsylvania each year. Unofficial counts indicate that this estimate is much too low. The probable number of migrants and dependents is nearer 16,000. This does not

Dr. Chapman is director of the bureau of planning, evaluation, and research, Pennsylvania Department of Health, Harrisburg. This paper is based on a speech presented at the Thirteenth Annual Pennsylvania Health Conference, Pennsylvania State University, University Park, on August 18, 1964. include the number of in-State migrants who use cities like Philadelphia as their base.

One of the major streams of migrants moves up the Atlantic coast. For the most part, they are Negroes from Florida plus other Negroes, recruited as the need arises, from cities and towns along the way north. Added to these are some Puerto Ricans who come to Pennsylvania.

Migrants travel in crews of 20 to 300 . A crew leader recruits them, supplies transportation, obtains work, arranges for housing, and, depending on his scruples, may or may not provide other services for a fee.

As in any cross section of people, the ethics of crew leaders vary. Most are honest and have the best interest of their members at heart. Others are dishonest and exploit them in many ways.

\section{Problems of Migrants}

Most Americans today have a family, own or rent a comfortable home, have access to educational facilities, are privileged to go to the church of their choice, and take many other advantages for granted that are not within the migrant's reach.

Mobility. Most people enjoy camping out for short periods of time, but few enjoy living that way-as migrants do-the best part of each year.

Moving around as migrants do, the education of their children is constantly interrupted. In most cases, it is soon abandoned as hopeless. Children are brought up with few normal social contacts. They have only limited access to community facilities and to other people. 
Income. In 1957 the average migrant worker earned $\$ 859$ per year. The average family's earnings were less than $\$ 1,800$ and this income varied from month to month.

Housing. In Pennsylvania housing for migrants has improved steadily over the last decade. However, many housing units are still poorly maintained and dilapidated. Toilet facilities, perhaps adequate at the beginning of the season, tend to suffer from poor maintenance as the season wanes. Overcrowding, although relative, is common.

Health. Migrants receive little benefit from the preventive health and medical services available to the general population. Health services consist of taking care of their diseases, disabilities, and emergencies as they arise. There has been no organized system of care except for children. Physicians in many communities have cared for migrants, often at great personal sacrifice. Too much dependence has been placed on and too much has been asked of the relatively few doctors who have generously offered their services.

However, in some areas, and increasingly at their home base, preventive services are being augmented. In Pennsylvania migrants may be hospitalized by the department of public welfare for pregnancy, surgery, and acute medical conditions.

Migrants generally share the health problems of other families that have been handicapped by poverty, minority group status, ignorance, and social isolation. Poor nutrition, bad teeth, diarrheal diseases, intestinal parasites, venereal disease, respiratory ailments, muscle strain, and accidental injuries are typical reasons why they seek medical care.

A pilot project conducted in 1963 at the Geisinger Medical Center in Danville, Pa., indicated that one of the basic needs of migrants was the development of family health clinics to which migrant workers and members of their families could go for the treatment of illnesses normally treated by general practitioners.

In this study, covering a four-county area in the central part of the State, 187 migrants or family members were seen by physicians. Among this group 270 conditions were found that warranted medical or nursing attention. A breakdown of conditions follows:

\section{Condition}

Number of

Infective and parasitic diseases

cases

Allergic, metabolic, and nutritional diseases -- 12

Diseases of the nervous system__________-_ 21

Diseases of the circulatory system_._._._.- 16

Diseases of respiratory system__-______-_- 25

Diseases of the genitourinary system____._- 16

Diseases of digestive tract._._._._-_._- 7

Diseases of the skin and cellular tissue_._-_- 10

Diseases of bones_._-_-_- 15

Pregnancy -...

Diabetes --.--

Symptoms and ill-defined conditions___._._._. 34

Other findings___._-_._- 71

Mental health. Very little is known about the mental health of migrants. The drunkenness and outbreaks of violence reported from the camps are, as a rule, dismissed with only a shrug. However, drunkenness and violence may be a predictable byproduct of the frustrations of a group that has been consistently barred from the rewards and opportunities normally available to permanent residents of communities.

\section{Conclusions}

In Pennsylvania migrant workers are essential for harvesting crops which have a large monetary value and are important to the State's economic health. These workers and their families have a lower than average income, are poorly educated, and have little opportunity to participate in normal community activities.

Most migrants do not receive preventive health care on a continuing basis. Their emergency medical needs are met as they arise in a variety of ways. Much still needs to be done to develop a systematic way of providing preventive health services to migrants, but this need extends to other groups in most communities as well.

Progress is being made in improving housing and sanitation in migrant camps. The day may come when migrant housing will be centralized on a county or regional basis, which will simplify camp management. It also would provide a convenient place at which to hold clinics, maintain day care centers, and provide similar types of services.

Knowledge gained in working with migrants will be useful in understanding the health needs of other underprivileged groups in the population. 


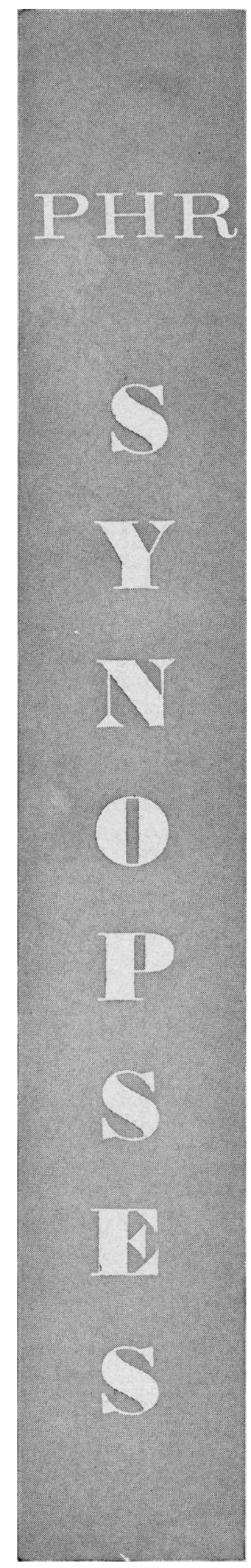

HALPERT, HAROLD P. (Public Health Service): Surveys of public opinions and attitudes about mental illness: Implications for communications activities. Public Health Reports, Vol. 80, July 1965, pp. 589-597.

Surveys of public opinions and attitudes conducted during the past 15 years demonstrate increasing public understanding of mental illness and greater acceptance of the mentally ill. Findings indicate that younger people, the better educated, and those in higher status occupations usually hold more enlightened opinions. A person's professional frame of reference may affect his attitudes about mental illness and how the mentally ill should be treated. The degree of exposure to information about mental illness and to psychiatric patients also is an important factor, sometimes more important than formal education, in determining degree of enlightenment.

Several important implications for programing communications activities emerge from these studies of public opinions and attitudes: People need to be told where to go for help with emotional problems. Special educational programs must be developed for persons in population subgroups, where it still is considered shameful to seek psychiatric help. People need to be exposed to information about mental health and mental illness many times and in many different ways. Educational activities should have well-defined, concrete, purposeful objectives, and should be geared to the prospective audience's frame of reference. Before undertaking to teach people about mental health and mental illness, it is most important to find out what they want to know and what they need to know.

ROSENBLUM, MARCUS (Public Health Service), ET AL.: Needs of a drug information system. Hospital pharmacists' conference. Public Health Reports, Vol. 80, July 1965, pages 603-614.

The experiences of pioneer hospitalbased drug information centers, as described by participants in a seminar sponsored by the Public Health Service and the American Society of Hospital Pharmacists, are related to the needs of users of a prospective system and a na- tional center for drug information. The report of the seminar is supplemented by comments of individual participants. One of the major conclusions cited was that a drug information system will thrive or wither according to the support it receives from leading physicians.

Baganz, PaUl C. (Public Health Service Hospital, Fort Worth, Tex.), and MADDUX, JAMES F.: Employment status of narcotic addicts one year after hospital discharge. Public Health Reports, Vol. 80, July 1965, pages 615-621.

Information about employment status 1 year after discharge from the Public Health Service Hospital, Fort Worth, Tex., of 100 former narcotic addict patients was obtained from their probation officers. The patients had compulsory supervision for at least 1 year following discharge. Sixty-three were reported to be employed, and six of these were engaged in skilled occupations which they had learned in the hospital. Thirty percent of the unemployed were in further legal difficulty.

Years of education was positively cor- related with posthospital employment. Completion of high school was also correlated with employment, but the correlation was statistically significant only when 14 patients who had earned high school equivalency certificates during hospitalization were counted as having completed high school. Use of psychotherapy or counseling during hospitalization was positively correlated with employment. Principal work assignment in a production line industry with pay during hospitalization was negatively correlated with employment. 


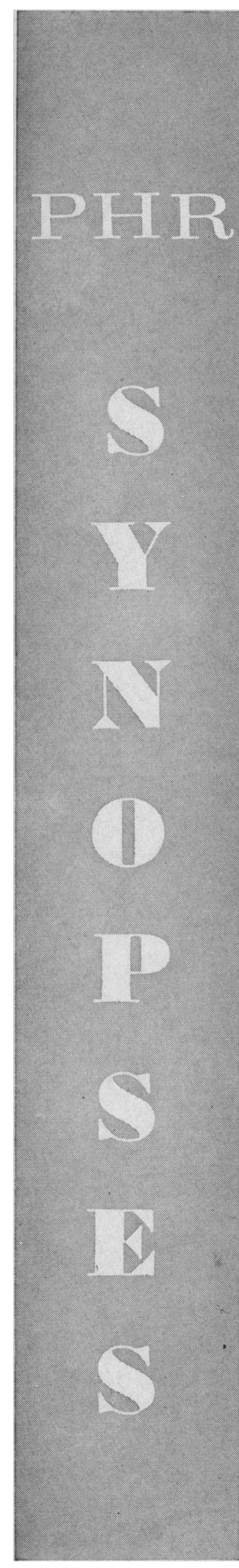

ROSNER, LESTER J. (New York City Department of Health) : Applications of automatic data processing to a public health agency's operations. Public Health Reports, Vol. 80, July 1965, pp. 625-632.

The New York City Health Department has had experience with mechanical data processing equipment for 70 years. Until fairly recently, the agency used automatic data processing systems chiefly to compile service data and statistics on communicable diseases and in recording, processing, and tabulating births and deaths. More recently, ADP systems have been developed and successfully installed in the administrative and business management operations of the department. The ADP systems outlined in the paper include: (a) an automated system for issuance and renewal of permits and licenses for businesses and establishments under the department's jurisdiction; (b) a system to monitor and control inspections of retail and wholesale food establishments that operate under permits issued by the department; (c) an application of ADP to the purchasing of supplies and materials; and $(d)$ the use of ADP and a computer in operations of a community blood bank.

GRANT, MURRAY (District of Columbia Department of Public Health): Screening for chronic disease with a mobile health unit. Public Health Reports, Vol. 80, July 1965, pp. 633-636.

During the year April 23, 1963-April 30,1964 , a total of 7,269 persons over 40 years of age were screened by a mobile health unit at 3 locations in the District of Columbia.

Of this number 5,801 were referred because of some apparent difficulty and for 2,658 of these, followup was completed within a year. The median age of screenees was 51.9 years. The most common abnormalities were high blood pressure, obesity, abnormal electrocardiogram, loss of hearing, and suspicion of glaucoma. Approximately 75 percent of persons screened and found to have an apparent abnormality were seen by their private physicians; the remainder were sent to public clinics.

Health educators were responsible for persuading persons to visit the specially equipped bus and chronic disease advisers followed patients through to definitive diagnosis.

BARKER; ANNA E. (Kentucky Mental Health Manpower Commission, Louisville), and STATON, EARL E.: Inactive nurses. An untapped recruitment source. Public Health Reports, Vol. 80, July 1965, pp. 637-645.

In a 1963 study of nurses registered as inactive in Kentucky, the Kentucky Mental Health Manpower Commission used a questionnaire and followup survey to obtain data on the nurses' family status, educational history, work record, and other related factors. The study's purpose was to determine why nurses are inactive, the extent to which they may be expected to return to nursing employment, and to seek new methods to ease the shortage of nurses in mental health facilities.

Results of the study revealed that approximately one of every two inactive nurses who maintain their registration in Kentucky may eventually return to nursing. Generally, the nurse who will re-enter the field is under 50 years of age, has one to three children, and has received her training fairly recently. The nurse who plans to return prefers a part-time staff position in a hospital setting. Previous psychiatric work experience or formal psychiatric training seems to have little effect on the selection of psychiatric nursing as a specialty. Lack of available care for children is a major obstacle to the nurses' re-entry into the field. 


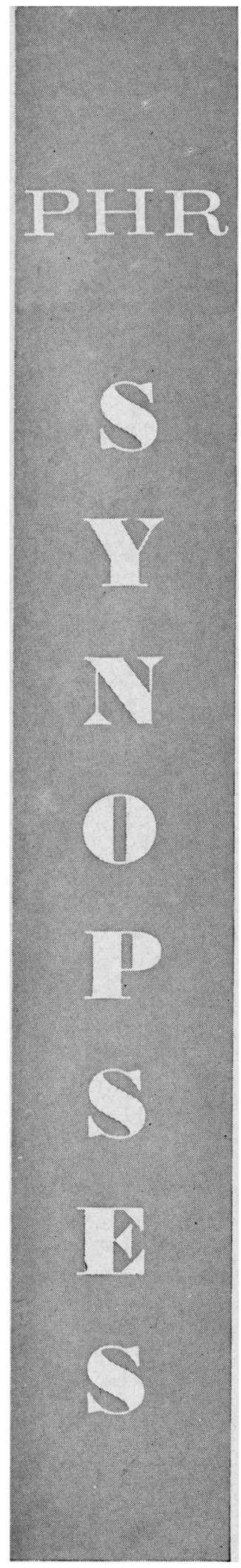

LUCAS, JAMES B. (Public Health Service), JOHnston, J. GIBSON, KAYE, HAROLD S., BUCCA, MATTHEW A., and ROBINSON, ROSLYN Q.: Production of adenovirus antiserums in horses. Public Health Reports, Vol. 80, July 1965, pp. 647-652.

Studies were conducted to determine the suitability of using horses for the economical large-volume production of adenovirus antiserums. Antiserums were produced with adenovirus types 1 through 18. Methods of antigen production and control and immunization schedules are described. All serums were tested for homologous and heterotypic crosses in serum neutralization and hemagglutination inhibition tests. Most serums were found to be satisfactory for use as reference or working reagents for the identification of adenovirus isolates with either the serum neutralization or hemagglutination inhibition tests or both. Generally, serums were found to be less specific in the hemagglutination inhibition test, and greater variability with repeated testing was noted.
The extent of heterologous cross reactivity among these 18 serotypes is reported, and suggestions are made that might minimize them in future production schedules. Studies with adenovirus types 3, 7a, and 14 disclosed that hemagglutination inhibition serum titers were not consistently reproducible with the same antigen preparation but different lots of rhesus erythrocytes. This variability in erythrocyte sensitivity causes a variation in antigen requirement for separate hemagglutination inhibition tests, which directly affects the titer obtained for the serum. For this reason it has been suggested that, with these three serotypes, a precise definition of serum titers should be expressed in terms of the hemagglutinin titration.

GITHENS, JOHN H. (University of Colorado Medical Center), ELLIOT, F. EILEEN, and SAUNDERS, LYLE H.: The relation of socioeconomic factors to incidence of childhood leukemia. Public Health Reports, Vol. 80, July 1965, pp. 573-578.

The relationship between certain social and economic factors and the incidence of childhood leukemia in Colorado was studied for the 19-year period from 1941 through 1959. Identifying information was obtained from death certificates of 258 children, and U.S. Bureau of the Census reports were used to obtain data on the social and economic backgrounds and population.

The rate of reported cases of childhood leukemia in the State increased from 1941 to 1959. The last 5 years (1955-59) showed a 2.7-fold increase over the first 5 years $(1941-45)$ of this period.

The leukemia death rate appeared to be about $21 / 2$ times higher for urban children than for those living in rural Colorado. In the large urban area of Denver, the incidence of leukemia was two to almost four times higher in children living in the census tracts that were socially and economically more favored compared with children of low-income families from census tracts with the poorest housing conditions. No single social factor among those studied showed a signifcantly higher correlation than the others.

A consistent relationship of childhood leukemia with these social and economic factors was found by various methods of analysis. Although the true significance of these differences is difficult to determine, a number of possible etiologic factors might explain such a pattern. These include the genetic or ethnic background of the children, the influence of variations in diet and in exposure to various physical agents such as $X$-rays, and differences in exposure to infections including enteric viruses.

The nature of a paper, not its importance or significance, determines whether a synopsis is printed. See "Information for Contributors" on next page. 